\title{
Stoichiometry and kinetics of single and mixed substrate uptake in Aspergillus niger
}

\author{
Francisca Lameiras $^{1} \cdot$ Cor Ras $^{1} \cdot$ Angela ten Pierick $^{1} \cdot$ Joseph J. Heijnen $^{1}$. \\ Walter M. van Gulik ${ }^{1}$
}

Received: 3 March 2017 / Accepted: 11 October 2017 / Published online: 19 October 2017

(C) The Author(s) 2017. This article is an open access publication

\begin{abstract}
In its natural environment, the filamentous fungus Aspergillus niger grows on decaying fruits and plant material, thereby enzymatically degrading the lignocellulosic constituents (lignin, cellulose, hemicellulose, and pectin) into a mixture of mono- and oligosaccharides. To investigate the kinetics and stoichiometry of growth of this fungus on lignocellulosic sugars, we carried out batch cultivations on six representative monosaccharides (glucose, xylose, mannose, rhamnose, arabinose, and galacturonic acid) and a mixture of these. Growth on these substrates was characterized in terms of biomass yields, oxygen/biomass ratios, and specific conversion rates. Interestingly, in combination, some of the carbon sources were consumed simultaneously and some sequentially. With a previously developed protocol, a sequential chemostat cultivation experiment was performed on a feed mixture of the six substrates. We found that the uptake of glucose, xylose, and mannose could be described with a Michaelis-Menten-type kinetics; however, these carbon sources seem to be competing for the same transport systems, while the uptake of arabinose, galacturonic acid, and rhamnose appeared to be repressed by the presence of other substrates.
\end{abstract}

Keywords Aspergillus niger . Plant waste streams . Substrate uptake $\cdot$ Kinetics $\cdot$ Stoichiometry

Electronic supplementary material The online version of this article (doi:10.1007/s00449-017-1854-3) contains supplementary material, which is available to authorized users.

\footnotetext{
Walter M. van Gulik

w.m.vangulik@tudelft.nl

1 Cell Systems Engineering section, Department of Biotechnology, Delft University of Technology, 2629 HZ Delft, The Netherlands
}

\section{Introduction}

In white biotechnology, the feedstock represents the highest cost factor in the production of bulk chemicals. Therefore, there is an increasing interest in using cheaper biomass streams as feedstock for industrial biotechnology processes, the so-called second-generation feedstocks.

The second-generation feedstocks have the advantage that they do not compete with food supplies, but have the disadvantage that they are much more complex than firstgeneration ones. The second-generation feedstocks consist of mixtures of different fermentable carbon sources from plant biomass of agricultural crops waste, which are currently insufficiently used. Of the global $200 \times 10^{9}$ tons per year of plant biomass produced, over $90 \%$ is lignocellulose. About $8-20 \times 10^{9}$ tons of this biomass is potentially accessible, but remains unexploited [1].

In [2], the performance of six industrially relevant microorganisms was tested by submitting them to growth conditions that they encounter in a second-generation feedstockbased production process (mixture of substrates, inhibitors, extreme $\mathrm{pH}$, etc.). The generated data were used to rank the organisms by relative performance, and Aspergillus niger scored the highest, leading to a stronger motivation for the use of this microorganism in the second-generation feedstocks [2].

A. niger is known to grow on hexose as well as pentose substrates, and can also hydrolyse plant material due to high levels of excreted enzymes having the capacity to degrade the plant cell wall polysaccharides [3].

Lignocellulose contains three structural polysaccharides: cellulose (40-50\%), hemicellulose (25-35\%), and lignin (15-20\%) [4]. Besides glucose, sugar monomers in hemicellulose can include xylose, galactose, mannose, and 
arabinose; pectins on the other hand are rich in galacturonic acid and rhamnose.

The shift of industrial biotechnology from highly refined sugar syrups (first-generation feedstocks) to more sustainable and cheaper carbon and energy sources requires a shift from single substrate to mixed substrate use [5].

To obtain a better understanding of the uptake of individual substrates in a complex mixture as well as the stoichiometry and kinetics of growth and product formation under those conditions, careful kinetic studies are required. In general, the uptake and metabolism of different carbon sources in heterotrophic microorganisms are controlled through carbon catabolite repression (CCR). In many cases, glucose is the preferred substrate for growth, and thus, the presence of glucose will repress the uptake and metabolism of other available carbon sources. Glucose repression was first studied in the bacterium Escherichia coli [6] and later on also in yeasts and filamentous fungi [7].

In this work, we studied the capacity of A. niger for uptake of six-selected monosaccharides present in lignocellulosic biomass, that is, three C6 sugars (glucose, mannose, and rhamnose) two C5 sugars (xylose and arabinose), and a sugar acid (galacturonic acid). We investigated the stoichiometry and kinetics of substrate uptake and aerobic growth on these different carbon sources, under substrate limited (sequential chemostat cultures) as well as substrate excess conditions (shake flask and batch cultivations), using single and mixed substrate feedstocks. With the application of $A$. niger for the production of organic acids in mind, we used a strain (A. niger NW185) not capable of producing gluconic and oxalic acid and carried out all cultivations at $\mathrm{pH} 2.5$.

\section{Materials and methods}

\section{Strain and inoculum}

The strain used was A. niger NW185 (cspAl short conidiospores, fwAl fawn coloured spores, gox $C 17$ glucose oxidase negative, and prtF 28 oxalate non-producing). Unless stated otherwise, the hyphal inocula for batch and continuous cultivations were obtained from shake flask cultures. Spores for pre-inoculum were grown on agar plates and harvested, as described in [8]. A concentration of $1 \times 10^{6}$ spores $/ \mathrm{mL}$ was pre-inoculated in a $250 \mathrm{~mL}$ shake flask containing $100 \mathrm{~mL}$ of $\mathrm{PM}$ medium and $100 \mathrm{mM}$ of sorbitol [9] at $\mathrm{pH}$ 4.5. Sorbitol was used as a pre-substrate for the pre-cultivation as it does not induce or repress any catabolic system [10].

The shake flask was incubated overnight at $30{ }^{\circ} \mathrm{C}$ in an orbital shaker (CERTOMAT BS-1, Sartorius group) at $250 \mathrm{rpm}$. The resulting mycelia suspension was separated by centrifugation (Heraeus Biofuge Stratos, Thermo Scientific, USA) for $2 \mathrm{~min}$ at $10000 \mathrm{G}, 4^{\circ} \mathrm{C}$ and washed two times with
$50 \mathrm{~mL}$ PM medium without carbon source at room temperature and subsequently used to inoculate the bioreactor (one shake flask per bioreactor).

\section{Shake flask cultivation}

A. niger was inoculated in $250 \mathrm{~mL}$ shake flasks, containing $100 \mathrm{~mL}$ minimal medium $\left[0.5 \mathrm{~g} / \mathrm{L}\left(\mathrm{NH}_{4}\right)_{2} \mathrm{SO}_{4}, 0.3 \mathrm{~g} / \mathrm{L}\right.$ $\mathrm{KH}_{2} \mathrm{PO}_{4}, 3 \mathrm{~g} / \mathrm{L} \mathrm{NH}_{4} \mathrm{H}_{2} \mathrm{PO}_{4}$ and $\left.0.5 \mathrm{~g} / \mathrm{L} \mathrm{MgSO}_{4} \cdot 7 \mathrm{H}_{2} \mathrm{O}\right]$, at initial $\mathrm{pH} 2.5$. The $\mathrm{pH} 2.5$ for growth in continuous cultivation has high advantages in terms of morphology and broth homogeneity [8], but is also highly relevant because we are interested in organic acid production.

In each shake flask, the medium was supplemented with one single carbon source, such that the added amount of carbon was the same (total $228 \mathrm{mCmol} / \mathrm{L}$ ), namely, $38 \mathrm{mM}$ of D-glucose, D-galacturonic acid, L-rhamnose and D-mannose, and $45.6 \mathrm{mM}$ of D-xylose and L-arabinose. Furthermore, $1 \mathrm{~mL} / \mathrm{L}$ of trace elements solution was added, containing $10 \mathrm{~g} / \mathrm{L}$ EDTA, $4.4 \mathrm{~g} / \mathrm{L} \mathrm{ZnSO}{ }_{4} \cdot 7 \mathrm{H}_{2} \mathrm{O}, 1.0 \mathrm{~g} / \mathrm{L} \mathrm{MnCl}{ }_{2} \cdot 4 \mathrm{H}_{2} \mathrm{O}$, $0.32 \mathrm{~g} / \mathrm{L} \mathrm{CoCl} \cdot 6 \mathrm{H}_{2} \mathrm{O}, 0.32 \mathrm{~g} / \mathrm{L} \mathrm{CuSO}{ }_{4} \cdot 5 \mathrm{H}_{2} \mathrm{O}, 0.22 \mathrm{~g} / \mathrm{L}$ $\left(\mathrm{NH}_{4}\right)_{6} \mathrm{Mo}_{7} \mathrm{O}_{24} \cdot 4 \mathrm{H}_{2} \mathrm{O}, 1.47 \mathrm{~g} / \mathrm{L} \mathrm{CaCl} \cdot 2 \mathrm{H}_{2} \mathrm{O}$, and $1.0 \mathrm{~g} / \mathrm{L}$ $\mathrm{FeSO}_{4} \cdot 7 \mathrm{H}_{2} \mathrm{O}$ [11].

Medium was sterilized at $121{ }^{\circ} \mathrm{C}$ for $20 \mathrm{~min}$ and the carbon-source solutions were sterilized separately at $110^{\circ} \mathrm{C}$. The trace elements were added sterile to the culture media by filtration through a $0.2 \mu \mathrm{m}$ cartridge filter (Whatman FP 30/0.2 CA-S).

One set of shake flasks was inoculated with spores and the second set with washed mycelia from one shake flask previously grown in PM medium as described above.

Cultures were incubated for $50 \mathrm{~h}$ in an orbital shaker at $30{ }^{\circ} \mathrm{C}$ and $250 \mathrm{rpm}$ (CERTOMAT BS-1, Sartorius group).

\section{Single carbon-source batch cultivation}

Batch cultivations were operated in a $2 \mathrm{~L}$ bioreactor with $1 \mathrm{~L}$ working volume (Applikon, Schiedam, The Netherlands), on minimal medium (see previous section) supplemented with either $38 \mathrm{mM}$ D-glucose, $38 \mathrm{mM}$ D-galacturonic acid, $38 \mathrm{mM}$ L-rhamnose, $38 \mathrm{mM}$ D-mannose, $45.6 \mathrm{mM}$ of D-xylose or $45.6 \mathrm{mM}$ L-arabinose.

The bioreactor was equipped with $\mathrm{pH}$, temperature, and dissolved oxygen probes. Throughout the cultivation, the $\mathrm{pH}$ was kept at $2.5( \pm 0.1)$ by controlled addition of $1 \mathrm{M} \mathrm{NaOH}$ and the temperature was controlled at $30{ }^{\circ} \mathrm{C}( \pm 0.1)$.

Sterile air was supplied via a mass flow controller (Brooks 58505 calibration at $0{ }^{\circ} \mathrm{C}$ and 1 bar) at 0.22 vvm through a bottom sparger, and an overpressure of 0.3 bar was applied.

The exhaust gas of the fermentor was passed through a condenser $\left(4^{\circ} \mathrm{C}\right)$ and a Nafion dryer (Permapure, Toms River, USA) before entering an NGA 2000 offgas analyser (Rosemount Analytical, Anaheim, USA) for measurement 
of the oxygen and carbon dioxide contents. Data acquisition was performed with MFCS/win 3.0 software.

\section{Mixture of carbon-source batch cultivation}

Fermentation conditions and control were executed as described for the single substrate batch fermentations (previous section), with the difference that the minimal medium was supplemented with equimolar concentrations of carbon of six different carbon sources $(6.3 \mathrm{mM}$ D-glucose, $6.3 \mathrm{mM}$ D-galacturonic acid, $6.3 \mathrm{mM}$ L-rhamnose, $6.3 \mathrm{mM}$ D-mannose, $7.6 \mathrm{mM}$ of D-xylose, and $7.6 \mathrm{mM} \mathrm{L}$-arabinose), to a total of $228 \mathrm{mCmol} / \mathrm{L}$.

\section{Sequential chemostat cultures}

In contrast to the batch fermentations (previous two sections), the sequential chemostat culture experiment was performed in a $7 \mathrm{~L}$ bioreactor with a working volume of $4.5 \mathrm{~L}$ Fermentor set up and operating conditions were the same, as described in [8], and temperature and $\mathrm{pH}$ were the same as the batch experiments.

The reactor was inoculated with pre-grown and washed mycelia into minimal media supplemented with $12.6 \mathrm{mM}$ D-glucose, $12.6 \mathrm{mM}$ D-galacturonic acid, $12.6 \mathrm{mM}$ L-rhamnose, $12.6 \mathrm{mM}$ D-mannose, $15.2 \mathrm{mM}$ of D-xylose, and $15.2 \mathrm{mM}$ L-arabinose. After the batch phase was finished, the feed was initiated with a starting dilution rate of $0.04 \mathrm{~h}^{-1}$. Subsequently, the dilution rate was increased to $0.21 \mathrm{~h}^{-1}$ in 11 steps $(0.04,0.06,0.08,0.09,0.11,0.13,0.14,0.16,0.17$, 0.19 , and $\left.0.21 \mathrm{~h}^{-1}\right)$.

\section{Sampling and analytical procedures}

During batch cultivations (both single and mixture of substrates), at least six samples (in triplicates) at different timepoints were taken for quantification of cell dry weight, biomass elemental composition, extracellular metabolites, and total organic carbon (TOC), during the exponential phase and at the end of the batch. During the sequential chemostat cultures experiment, triplicate samples were taken after two residence times, at each dilution rate.

Biomass dry weight was determined by filtration of $5 \mathrm{~mL}$ of broth over glass fiber filters (47 mm, type A/E, Pall, USA), subsequent washing with $10 \mathrm{~mL}$ of water and drying at $70{ }^{\circ} \mathrm{C}$ until constant weight. All samples were analysed in quadruplicate.

Biomass elemental composition was determined by combustion and subsequent gas analysis (carbon dioxide, water vapour, and nitrogen mass fractions), gas chromatography (oxygen), and ICP-MS (phosphorus and sulphur) (Energy Research Centre of the Netherlands).
Filtrate samples were obtained by quickly (within $3 \mathrm{~s}$ ) withdrawing $5 \mathrm{~mL}$ of broth, via the overpressure on the fermentor, into a syringe containing cooled steel beads $\left(-20{ }^{\circ} \mathrm{C}\right)$ to bring the sample temperature down to $0{ }^{\circ} \mathrm{C}$ [12]. The broth was then immediately pressed through a $0.45 \mu \mathrm{m}$ cartridge filter (Millex-HV durapore PVDF membrane) into a sampling vial, which was directly frozen in liquid nitrogen.

The total organic carbon content of filtrate samples was quantified with a total organic carbon analyser (type TOCL, Shimadzu, Kyoto, Japan). With this method, both total carbon (TC) and inorganic carbon (TIC) were measured. The latter representing the content of dissolved carbon dioxide and carbonic acid salts. Subtracting the inorganic carbon from the total carbon, yielded the TOC in filtrate.

The residual concentration of the different carbon sources was measured by different methods.

For the batch cultivation on single substrates, analyses were implemented by an HPLC method, using an $\mathrm{H}^{+}$exchange column at $60{ }^{\circ} \mathrm{C}$ (Bio-Rad HPX-87H $300 \times 7.8 \mathrm{~mm}$ ), employing phosphoric acid $1.5 \mathrm{mmol} / \mathrm{L}$ in Milli-Q water at $70{ }^{\circ} \mathrm{C}$ as mobile phase with a refractive index detection (Waters 2414; sens $=1024$; temp $=30^{\circ} \mathrm{C}$ ) and UV detection (Waters 2489; $210 \mathrm{~nm}$ ).

For the mixture of carbon sources, both GC-IDMS/MS and HPLC methods were used. The concentrations of the sugars measured by isotope dilution mass spectrometry (GCIDMS/MS) were according to the protocols of [13] with the following changes: temperature gradient was slower to have a better separation for xylose and arabinose and the temperature was increased with $5{ }^{\circ} \mathrm{C} / \mathrm{min}$ instead of $10^{\circ} \mathrm{C} / \mathrm{min}$. Glucose $^{13}$ xylose $^{13}$ mannose ${ }^{13}$ and arabinose ${ }^{13}$ were added as internal standards (IS) for the same ${ }^{12} \mathrm{C}$ compounds, while for rhamnose and galacturonic acid, glucuronic acid and fumarate were used as IS respectively. The HPLC method used for the same measurements was performed in an ionexchange column in lead $(\mathrm{Pb})$ form column at $85^{\circ} \mathrm{C}$ (BioRad HPX-87P300 $\times 7.8 \mathrm{~mm}$ ), employing milliQ water at $70{ }^{\circ} \mathrm{C}$ as mobile phase with a refractive index detection (Waters 2414; sens $=1024$; temp $=30^{\circ} \mathrm{C}$ ) and UV detection (Waters 2489; $210 \mathrm{~nm}$ ).

The samples from the sequential chemostat cultivation experiment were analysed with a Dionex ICS-5000 HPIC system with AS-AP sampler, SP pump, Carbopac PA-20 $3 \times 150 \mathrm{~mm}$ column and Aminotrap $3 \times 30 \mathrm{~mm}$ precolumn employing $2 \mathrm{mM} \mathrm{NaOH}$ isocratic (for $17.5 \mathrm{~min}$ followed by a cleaning step of $5 \mathrm{~min}$ with $200 \mathrm{mM}$ and equilibration for $15 \mathrm{~min}$ ), $20 \mathrm{mM} \mathrm{NaOH}$ isocratic (15 min followed by 5 min cleaning with $200 \mathrm{mM} \mathrm{NaOH}$ and equilibration for $15 \mathrm{~min}$ ) or gradient: $15 \mathrm{~min} 10 \mathrm{mM} \mathrm{NaOH}$ followed by a linear increase to $200 \mathrm{mM}$ in $15 \mathrm{~min}$, then a linear gradient in 20 min with $0.5 \mathrm{M}$ sodium acetate in $200 \mathrm{mM} \mathrm{NaOH}$ from 4 to $40 \% \mathrm{v} / \mathrm{v}$, followed by a cleaning step of $5 \mathrm{~min} 200 \mathrm{mM}$ 
$\mathrm{NaOH}$ and equilibration for 15 min (for acidic sugars). All eluents with a flow of $0.5 \mathrm{~mL} / \mathrm{min}$ at $30{ }^{\circ} \mathrm{C}$. The detection was by electrochemical pulse at $15^{\circ} \mathrm{C}$.

\section{Balances, biomass specific rates, and data reconciliation during single carbon-source batch cultivation}

Using the gas-phase balances $\left(\mathrm{O}_{2}\right.$ and $\left.\mathrm{CO}_{2}\right)$ and liquid phase balances (biomass and substrate), the batch cumulative amounts of consumed $\mathrm{O}_{2}$ and substrate and produced $\mathrm{CO}_{2}$ and biomass were calculated.

Subsequently, because the carbon and degree of reduction balances were close to $100 \%$, the element reconciled cumulative amounts were calculated as function of time, as described in [14].

These reconciled amounts were used to calculate the maximum biomass specific conversion rates $\left(\mu^{\max }, q_{\mathrm{S}}{ }^{\max }\right.$, $q_{\mathrm{CO}_{2}}{ }^{\max }, q_{\mathrm{O}_{2}}{ }^{\text {max }}$, i.e., the biomass specific conversion rates of biomass, substrate, carbon dioxide, and oxygen, respectively) and the growth stoichiometric values.

The biomass elemental composition was analysed for each substrate and taken into account for the data reconciliation.

\section{Balances, biomass specific rates, and data reconciliation during sequential chemostat cultivation}

Using the steady-state mole balances, the rates of substrate $\left(R_{\mathrm{S}}\right)$, biomass $\left(R_{\mathrm{X}}\right)$, carbon dioxide $\left(q_{\mathrm{CO}_{2}}\right)$, oxygen $\left(q_{\mathrm{O}_{2}}\right)$, and by-products in the culture filtrate $\left(R_{\mathrm{TOC}}\right)$ were calculated from the primary measurements of concentrations in gas and liquid phases, as well as gas and liquid flow rates. From these rates, carbon and degree of reduction recoveries Eqs. (1), (2) were calculated:

Carbon recovery $(\%)=\frac{R_{\mathrm{x}}+R_{\mathrm{CO}_{2}}+R_{\mathrm{toc}}}{\sum R_{\mathrm{s}}} \times 100$

Degree of reduction(\%) $=\frac{\gamma_{x} R_{x}+\gamma_{\mathrm{toc}} R_{\mathrm{toc}}+\gamma_{\mathrm{O}_{2}} R_{\mathrm{O}_{2}}}{\Sigma \gamma_{\mathrm{S}} R_{\mathrm{s}}} \times 100$

where $\Sigma R_{\mathrm{S}}$ is the weighted sum of all consumed carbon sources in $\mathrm{Cmol} / \mathrm{L}$, and $R_{\mathrm{TOC}}$ is the residual total organic carbon resulted from the subtraction of the concentration of the residual substrates to the measured TOC. After checking absence of relevant carbon and degree of reduction gaps, data reconciliation was applied to obtain the best estimates of the measurements, within their error margins, using the elemental and charge conservation relations as constraints [15].

\section{Substrate uptake kinetics}

Microbial growth studies report several different equations to define substrate uptake kinetics. The most simple and widely used equations follow the hyperbolic Michaelis-Menten model of enzyme kinetics Eq. (3):

$q_{\mathrm{S}}=q_{\mathrm{S} \max } \times \frac{C_{\mathrm{S}}}{K_{\mathrm{S}}+C_{\mathrm{S}}}$

In a first approach of this study, the data obtained from the sequential chemostat cultivations were fitted to a Michaelis-Menten equation. When $C_{\mathrm{S}}=K_{\mathrm{S}}$, the value of qs corresponds to $50 \%$ of the $q_{\mathrm{S}}{ }^{\max }$.

Depending on the outcome of the fitting of Eq. (3), two situations were considered: competition for the same transport system or catabolite repression from other substrates.

To model the uptake of competing sugars, we assumed that these sugars are taken up by the same import systems and that their uptake could be described by competition, according to [16]-Eq. (4):

$v_{1}=\frac{v_{\max } S_{1}}{K_{m 1}\left(1+\sum_{i=2}^{n} \frac{S_{i}}{K_{m i}}\right)+S_{1}}$

where $S_{1}$ competes with $n-1$ substrates $S_{2}, \ldots, n$ for the binding site of the enzyme. The variables $K_{\mathrm{mi}}$ describe the Michaelis-Menten affinity constant for each subtract $S_{\mathrm{i}}$.

Catabolite repression of the uptake/metabolism of different carbon sources can be described by a so-called switch function, based on the Hill kinetics (Eq. 5), whereby the expression of an enzyme (e.g., transport protein or rate controlling enzyme of a catabolic pathway) is repressed by the presence of another carbon source:

$v_{\max }(R)=v_{\max }(U)\left(\frac{1}{1+\left(\frac{R}{K_{\mathrm{R}}}\right)^{n}}\right)$

Herein, $v_{\max }(R)$ and $v_{\max }(U)$ are, respectively, the repressed and unrepressed enzyme rates, $R$ is the repressor concentration, and $K_{\mathrm{R}}$ is the repression constant.

\section{Results and discussion}

\section{Shake flask cultivations on single carbon sources}

As a preliminary experiment to investigate which carbon sources would sustain growth and whether growth on different carbon sources would lead to different morphologies, A. niger was cultivated on minimal medium in shake flasks with six different substrates. Two sets of shake flasks cultivations were carried out. The first 
set was inoculated with spores, while the second set was inoculated with mycelia pre-grown on the same minimal medium with sorbitol as the carbon source (Fig. 1).

After $50 \mathrm{~h}$ of incubation, all shake flask cultures showed evident growth with the exception of rhamnose. L-Rhamnose did not seem to induce spore germination nor further growth of pre-grown mycelia. Nevertheless, growth has been observed on L-rhamnose as a sole carbon source for A. niger in minimal media [17, 18].

Further comparison between the sets of shake flask cultivations indicated that inoculation with spores generates heterogeneity in the morphology of the mycelia among the different carbon sources with sometimes the formation of pellets, while inoculation with mycelia induces a uniform and similar, pellet free, morphology for all the different carbon sources (Fig. 1). Such pellet free morphology is preferred in kinetic studies to avoid diffusion limitation.

\section{Bioreactor batch cultivations on single carbon sources}

To be able to study growth and substrate uptake of A. niger, batch cultures were performed on each single substrate, in $2 \mathrm{~L}$ bench scale bioreactors. All bioreactor cultures were inoculated with washed mycelia pre-grown on minimal media with sorbitol as the carbon source. During the batch cultivations, samples were taken for quantification of the concentrations of biomass and residual carbon source. The concentrations of $\mathrm{O}_{2}$ and $\mathrm{CO}_{2}$ in the offgas were measured online for the calculation of the $\mathrm{O}_{2}$ consumption and $\mathrm{CO}_{2}$ production rates as a function of time. The obtained profiles for the different batch cultures are shown in Fig. 2.

In contrast to what was observed for the shake flask cultivations, $A$. niger did grow on minimal media with rhamnose as sole carbon source in the bioreactor.

The first striking differences between the batch cultivations carried out on the different carbon sources were the duration of the lag phase and the total duration of the batch. The rhamnose cultivation had the longest lag phase (around $50 \mathrm{~h}$, and therefore explaining the absence of growth in shake flasks) and longest duration ( $85 \mathrm{~h}$ ). In contrast, the glucose, arabinose and mannose cultivations had short or no lag phases and were finished after about $30 \mathrm{~h}$.

It should be realized that the degree of reduction of the used carbon sources is not always the same [19]. For the sugars glucose, mannose, xylose, and arabinose, the generalized degree of reduction is four electrons per $\mathrm{Cmol}\left(\gamma_{\mathrm{s}}=4 \mathrm{e}^{-} / \mathrm{C}\right)$. However, galacturonic acid is more oxidized $\left(\gamma_{\mathrm{s}}=3.333 \mathrm{e}^{-} / \mathrm{C}\right)$ and rhamnose is more reduced $\left(\gamma_{\mathrm{s}}=4.333 \mathrm{e}^{-} / \mathrm{C}\right)$. This can clearly be seen from the respiratory quotients (Table 1 ), being the ratios of the biomass specific carbon dioxide production $\left(q_{\mathrm{C}}{ }^{\mathrm{max}}\right)$ and oxygen consumption $\left(q_{\mathrm{O}}{ }^{\max }\right)$ rates. As expected, for the substrates with a degree of reduction of $4 \mathrm{e}^{-} / \mathrm{C}$, the respiratory quotients have a value slightly higher than one; however, for the more oxidized substrate galacturonic acid, the value is significantly higher than one, while for the more reduced substrate rhamnose, the value is significantly lower than one.

The obtained measurements show in general that carbon and degree of reduction balances have recoveries of more than
Fig. 1 A. niger morphology after $50 \mathrm{~h}$ of growth in minimal media inoculated with spores (upper panel) and washed mycelia (lower panel). From left to right: glucose, xylose, arabinose, galacturonic acid, rhamnose, and mannose
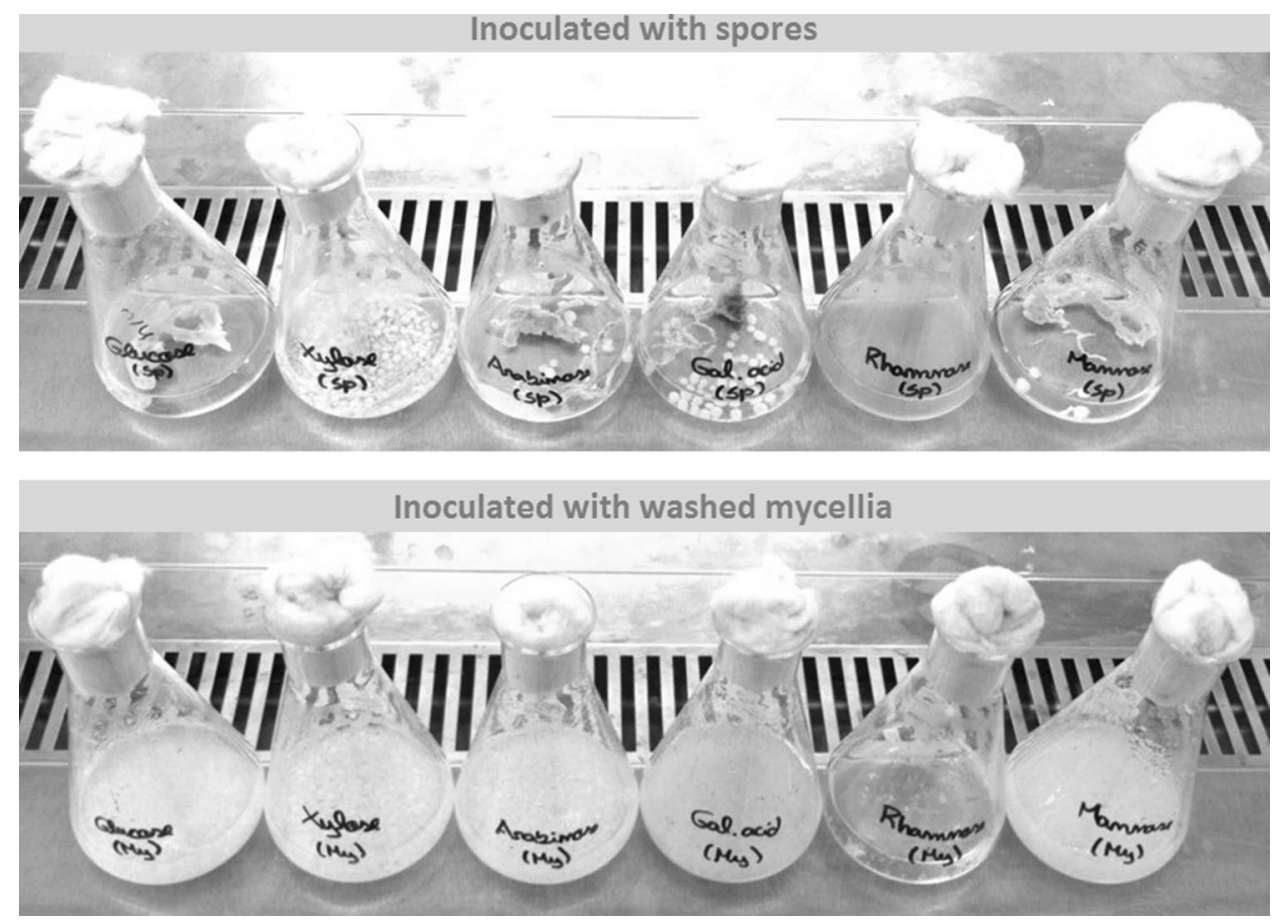


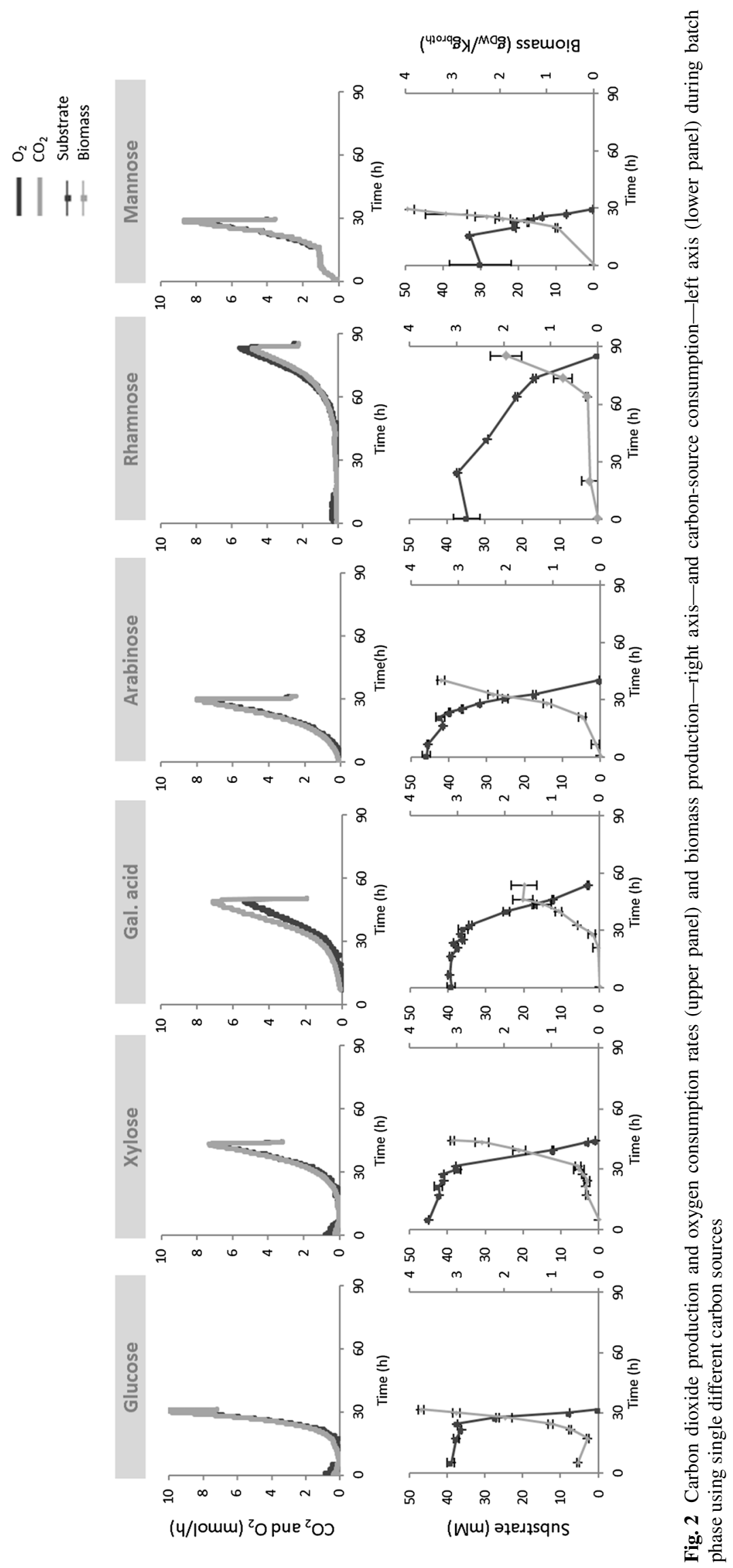


90\% (Table 1), indicating that by-product formation is either absent or insignificant.

The cumulative $\mathrm{O}_{2}, \mathrm{CO}_{2}$, substrate, and biomass data obtained during exponential growth (Online Resource 1) were used to obtain the best estimates of the biomass yield and biomass specific conversion rates (Table 1) using data reconciliation (explained in Materials and methods section). The biomass elemental composition was analysed for each substrate and taken into account for the data reconciliation (Online Resource 2).

Large differences in biomass yields and maximum biomass specific conversion rates were observed for the different single substrate cultivations. Glucose, xylose, and mannose were the substrates on which the highest biomass yield, maximum growth rate, and maximum substrate uptake rate were obtained.

From Table 1, the growth stoichiometry for unlimited exponential growth on the different substrates can be obtained by dividing the biomass specific conversion rates of substrate, oxygen, biomass (= maximum growth rate), and carbon dioxide by the maximum growth rate. This yields the overall reaction equations for the formation of $1 \mathrm{~mol}$ of biomass from substrate and oxygen:

-0.244 Glucose $-0.457 \mathrm{O}_{2}+1$ Biomass $+0.471 \mathrm{CO}_{2}$,

-0.262 Xylose $-0.295 \mathrm{O}_{2}+1$ Biomass $+0.302 \mathrm{CO}_{2}$,

-0.423 Arabinose $-1.113 \mathrm{O}_{2}+1$ Biomass $+1.124 \mathrm{CO}_{2}$,

-0.337 Galacturonic acid $-0.652 \mathrm{O}_{2}+1$ Biomass $+1 \mathrm{CO}_{2}$,

-0.245 Mannose $-0.477 \mathrm{O}_{2}+1$ Biomass $+0.477 \mathrm{CO}_{2}$,

-0.250 Rhamnose $-0.621 \mathrm{O}_{2}+1$ Biomass $+0.491 \mathrm{CO}_{2}$.
From an energy point of view, looking at the consumed oxygen per produced biomass, it is observed that xylose has the lowest oxygen consumption (about $0.30 \mathrm{~mol}_{\mathrm{O}_{2}} / \mathrm{mol}_{\text {biomass }}$ ). Glucose and mannose have a higher oxygen requirement (about $0.47 \mathrm{~mol}_{\mathrm{O}_{2}} / \mathrm{mol}_{\text {biomass }}$ ), while galacturonic acid and rhamnose have an even higher oxygen demand of respectively 0.65 and $0.62 \mathrm{~mol}_{\mathrm{O}_{2}} / \mathrm{mol}_{\text {biomass. }}$. Finally, arabinose has the highest oxygen consumption of $1.1 \mathrm{~mol}_{\mathrm{O}_{2}} / \mathrm{mol}_{\text {biomass }}$. These differences representing the differences in net energy expenditure for the formation of biomass for these different substrates may be due to different metabolic pathways. In case of arabinose, the energy and oxygen requirements are quite puzzling when compared to xylose, as this substrate has a very similar assimilation pathway in A. niger. These outputs ought to be further explored and confirmed by metabolic flux analysis.

\section{Bioreactor batch cultivations on a mixture of carbon sources}

The metabolic versatility of $A$. niger was investigated during growth on a mixture of all six carbon sources. Remarkably, in this cultivation, the $\mathrm{CO}_{2}$ profile resembled the typical pattern of diauxic growth (Fig. 3). This could indicate that some of the substrates were taken up and metabolised simultaneously, while the uptake and metabolism of other substrates were repressed and occurred only after the repressing substrates were depleted.

Quantification of the residual concentrations of the different carbon sources at different timepoints during the batch cultivation was carried out with two methods (HPLC and GC-IDMS/MS), which gave essentially the same results. Here, we only show the results obtained with GC-IDMS/MS. We plotted each substrate in a different graph to facilitate visualization (Fig. 3). From these plots, it can be seen that glucose consumption started immediately, while the other carbon sources were not consumed during the first $10 \mathrm{~h}$ after

Table 1 Carbon and degree of reduction recoveries

\begin{tabular}{|c|c|c|c|c|c|c|c|c|}
\hline $\begin{array}{l}\text { Carbon } \\
\text { source }\end{array}$ & $\begin{array}{l}\text { RQ respira- } \\
\text { tory quotient }\end{array}$ & $\begin{array}{l}\text { Carbon bal- } \\
\text { ance }(\%)\end{array}$ & $\begin{array}{l}\text { Degree of } \\
\text { reduction bal- } \\
\text { ance }(\%)\end{array}$ & $\begin{array}{l}Y_{\mathrm{X} / \mathrm{S}} \text { Biomass } \\
\text { yield }\left(\mathrm{Cmol}_{\mathrm{X}} /\right. \\
\left.\mathrm{Cmol}_{\mathrm{S}}\right)\end{array}$ & $\begin{array}{l}\mu^{\max } \max . \\
\text { growth rate } \\
\left(\mathrm{h}^{-1}\right)\end{array}$ & $\begin{array}{l}q_{\mathrm{S}}^{\max } \max \\
\text { substrate } \\
\text { uptake rate } \\
\left(\mathrm{mol}_{\mathrm{S}} \mathrm{h}^{-1}\right) / \\
\mathrm{Cmol}_{\mathrm{X})}\end{array}$ & $\begin{array}{l}q_{\mathrm{O}}{ }^{\max } \max . \\
\mathrm{O}_{2} \text { uptake rate } \\
\left(\mathrm{mol}_{\mathrm{O}_{2}} \mathrm{~h}^{-1}\right) / \\
\left(\mathrm{Cmol}_{\mathrm{X}}\right)\end{array}$ & 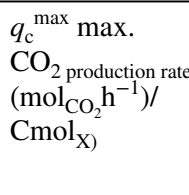 \\
\hline Glucose & $1.03 \pm 0.04$ & $94 \pm 2$ & $94 \pm 2$ & $0.68 \pm 0.03$ & $0.221 \pm 0.004$ & $0.054 \pm 0.001$ & $0.101 \pm 0.003$ & $0.104 \pm 0.003$ \\
\hline Xylose & $1.02 \pm 0.07$ & $87 \pm 2$ & $83 \pm 2$ & $0.77 \pm 0.06$ & $0.149 \pm 0.007$ & $0.039 \pm 0.002$ & $0.044 \pm 0.002$ & $0.045 \pm 0.002$ \\
\hline Arabinose & $1.01 \pm 0.04$ & $98 \pm 3$ & $94 \pm 5$ & $0.47 \pm 0.01$ & $0.097 \pm 0.001$ & $0.041 \pm 0.001$ & $0.108 \pm 0.003$ & $0.109 \pm 0.003$ \\
\hline Gal acid & $1.53 \pm 0.07$ & $93 \pm 4$ & $88 \pm 10$ & $0.50 \pm 0.06$ & $0.092 \pm 0.005$ & $0.031 \pm 0.001$ & $0.060 \pm 0.002$ & $0.092 \pm 0.003$ \\
\hline Mannose & $1.00 \pm 0.06$ & $96 \pm 2$ & $92 \pm 2$ & $0.68 \pm 0.03$ & $0.151 \pm 0.004$ & $0.037 \pm 0.001$ & $0.072 \pm 0.003$ & $0.072 \pm 0.003$ \\
\hline Rhamnose & $0.79 \pm 0.05$ & $101 \pm 5$ & $96 \pm 7$ & $0.67 \pm 0.03$ & $0.116 \pm 0.003$ & $0.029 \pm 0.001$ & $0.072 \pm 0.003$ & $0.057 \pm 0.003$ \\
\hline
\end{tabular}

Reconciled biomass yield and maximum biomass specific conversion rates for different carbon sources in A. niger in single batch cultivation 
Fig. 3 Carbon dioxide production rate and carbon source and biomass concentrations during batch phase in a mixture of six carbon sources
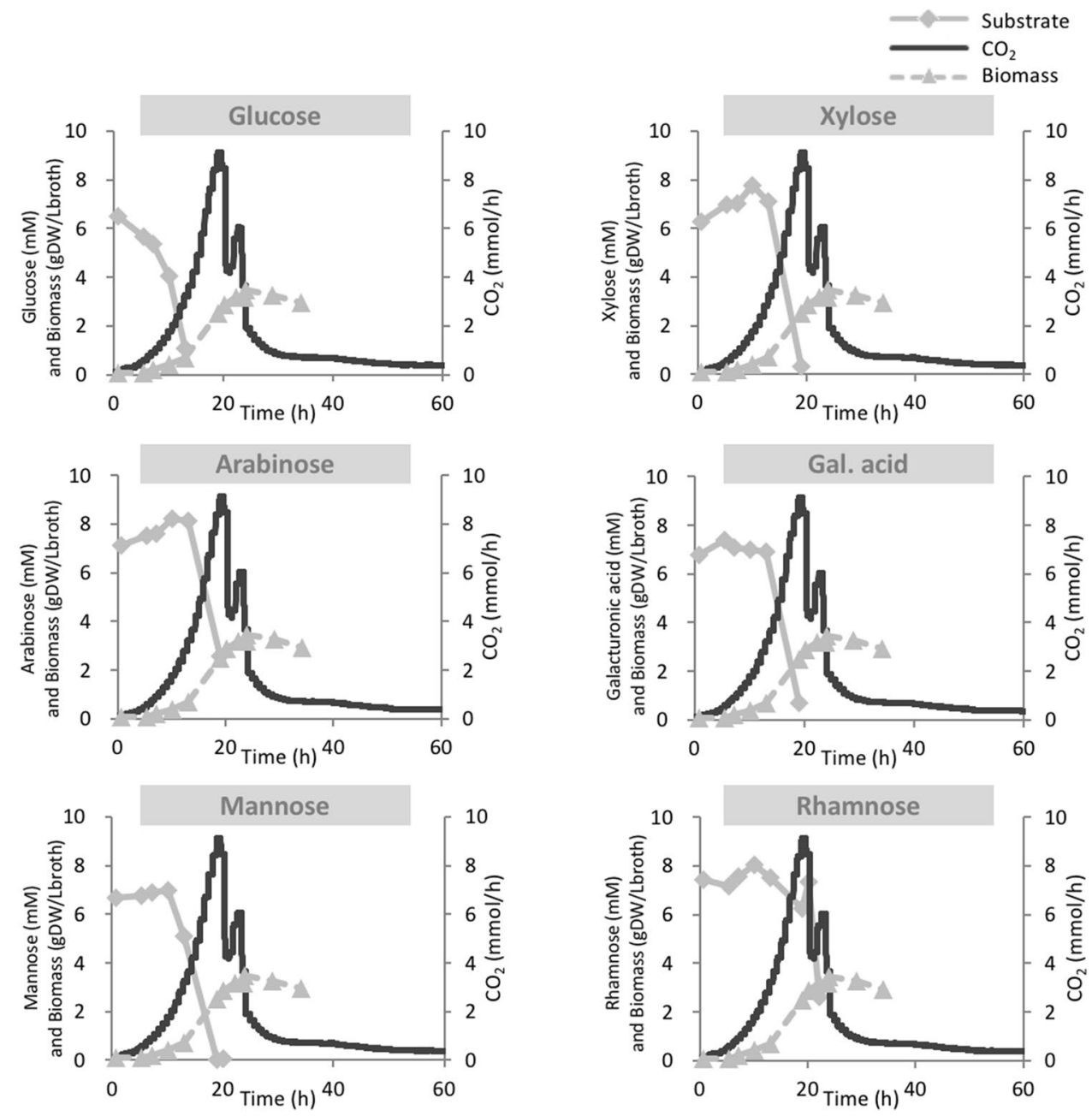

inoculation, indicating that the presence of glucose repressed the metabolism of all other carbon sources. The repression of the transport and metabolism of less preferred substrates in the abundance of a sugar which is easily metabolized, e.g., glucose, is a well-known phenomenon amongst microorganisms, also in case of filamentous fungi [20-22].

After glucose was almost depleted, mannose consumption started. At the sixth sample point, when mannose was depleted, consumption of arabinose, galacturonic acid and xylose occurred. Finally, rhamnose consumption started only after $20 \mathrm{~h}$ of cultivation when all other carbon sources were depleted, which coincided with a sharp decrease of the $\mathrm{O}_{2}$ consumption and $\mathrm{CO}_{2}$ production rates. In the period during which rhamnose was consumed, the respiration rate increased again for a short period of time and decreased sharply after depletion of this last substrate.

During the batch cultivation, the specific growth rate can be obtained from the online measurement of the respiration rate, that is either oxygen consumption or carbon dioxide production. During exponential growth, the respiration rate also increases exponentially, and thus, from a plot of the natural logarithm of, e.g., the $\mathrm{CO}_{2}$ production rate, the growth rate can be obtained. From this, it was calculated that during the initial $10 \mathrm{~h}$ of the batch cultivation; when glucose was the only carbon source consumed, the specific growth rate was equal to $0.264 \pm 0.005 \mathrm{~h}^{-1}$. Between 10 and $18 \mathrm{~h}$, during the consumption of mannose, arabinose, galacturonic acid, and xylose, the growth rate was $0.201 \pm 0.005 \mathrm{~h}^{-1}$ (Online Resource 3 ). The initial growth rate during the first $10 \mathrm{~h}$ on glucose was slightly higher than measured in the single substrate batch cultivation, while the growth rate obtained during consumption of mannose, arabinose, galacturonic acid, and xylose was significantly higher than the growth rates obtained from the single substrate batches (Table 1). This suggests that these carbon sources have individual uptake systems, which act in parallel.

\section{Sequential chemostat cultures}

To obtain a more detailed insight in the kinetics of substrate uptake in A. niger at low carbon-source concentrations, a carbon-limited sequential chemostat experiment was 
performed on a mixture of the six substrates used for the single and mixed substrate batch cultivations. The experiment was carried out in a $7 \mathrm{~L}$ bioreactor designed for homogeneous chemostat cultivation of A. niger, without pellet formation and/or wall growth [8]. The feed medium of the chemostat was minimal medium complemented with an equimolar mixture of the six carbon sources, as described in the materials and methods.

After achieving steady state, the dilution rate was increased stepwise and the chemostat was run until a new steady state was obtained. The initial dilution rate was $0.04 \mathrm{~h}^{-1}$ which was sufficiently lower than the lowest maximum growth rate determined for the single substrate batch cultivations (Table 1), to enable growth on all carbon sources in carbon-limited chemostat cultures.

For all dilution rates, measurements of the concentrations of residual substrates (Fig. 4), biomass dry weight, and oxygen and carbon dioxide levels (Fig. 5) were performed.
In addition, the total organic carbon (TOC) contents of the culture filtrate samples were determined. From these values, the measured amounts of residual substrates were subtracted to calculate the residual filtrate organic carbon, to verify whether by-products were formed (Online Resource 4). It was found that the in this way, determined residual carbon in the filtrate samples did not increase with increasing dilution rate, and was equal to an average value of $32 \pm 8 \mathrm{mCmol} / \mathrm{L}$, which was about $7 \%$ of the carbon supplied as mixed substrate to the culture. No attempts were undertaken to analyse the composition of the released carbon, as it was assumed that it mainly consisted of cell lysis products.

From the mentioned measurements (substrate, biomass, oxygen and carbon dioxide), it appeared that recoveries of carbon and degree of reduction were higher than $90 \%$ and thus the biomass specific rates were calculated and reconciled (Table 2, Online Resource 5).
Fig. 4 Residual concentration of carbon sources for different dilution rates during sequential chemostat cultures

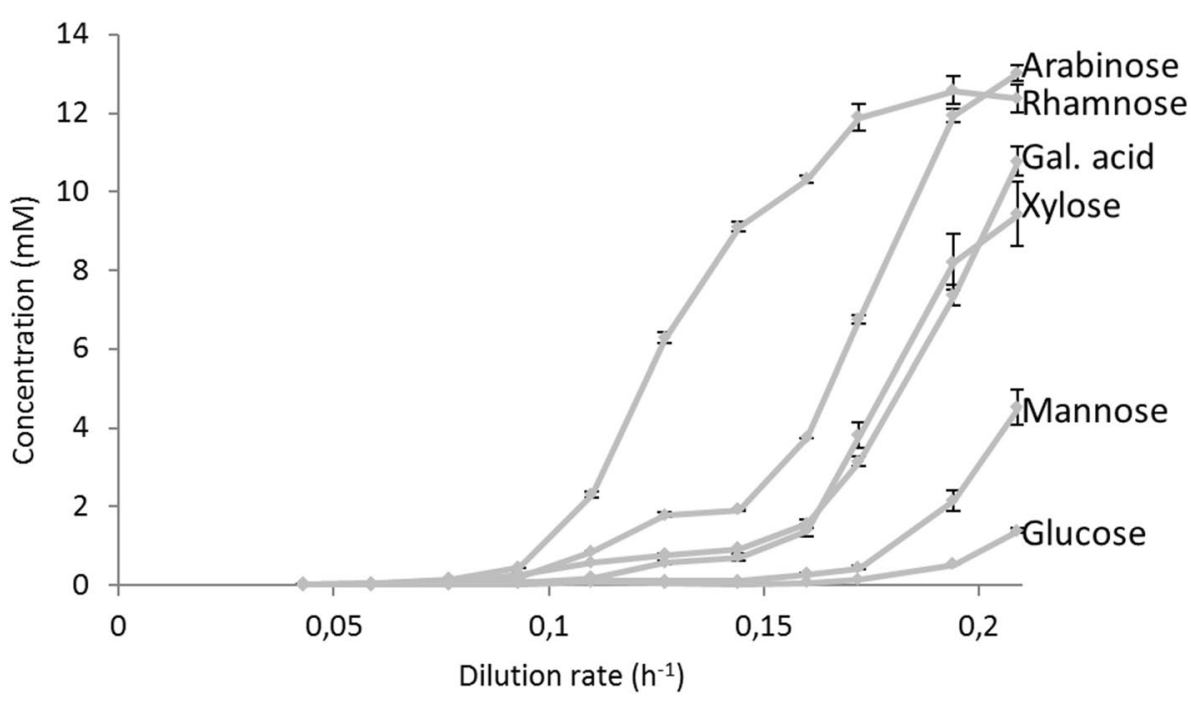

Fig. 5 Oxygen and carbon dioxide consumption/production rates, biomass concentration (closed square), and number of residence times $(\# \tau)$ during sequential chemostat cultivation

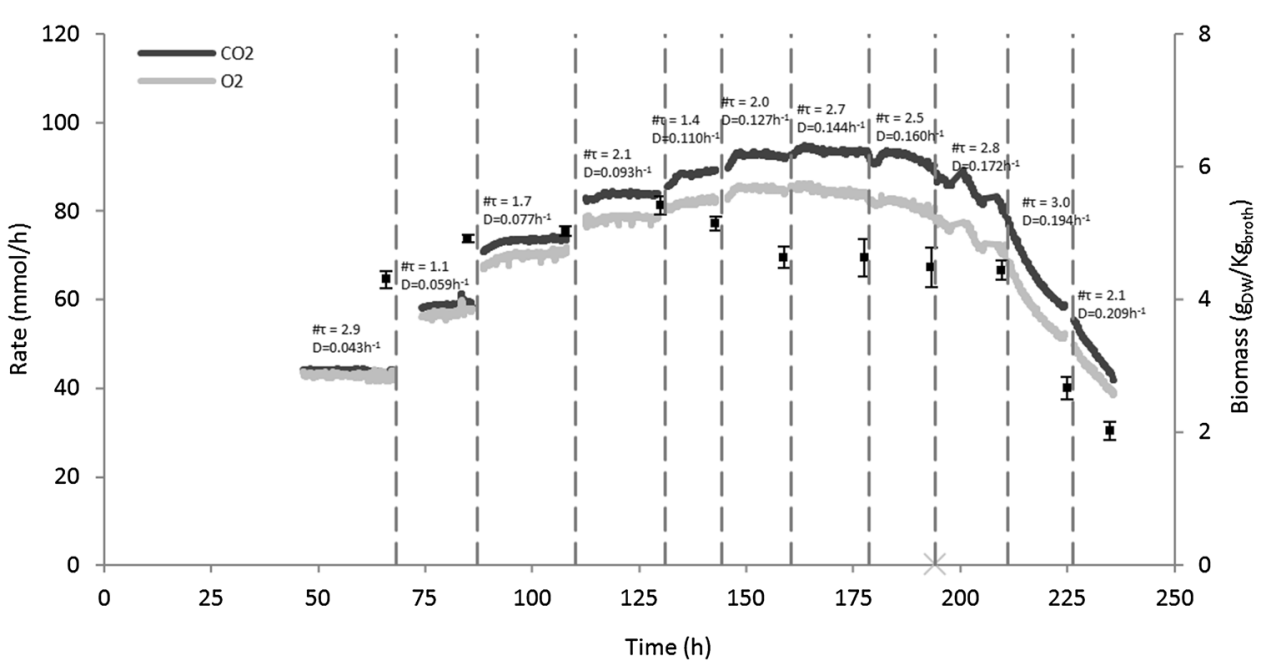




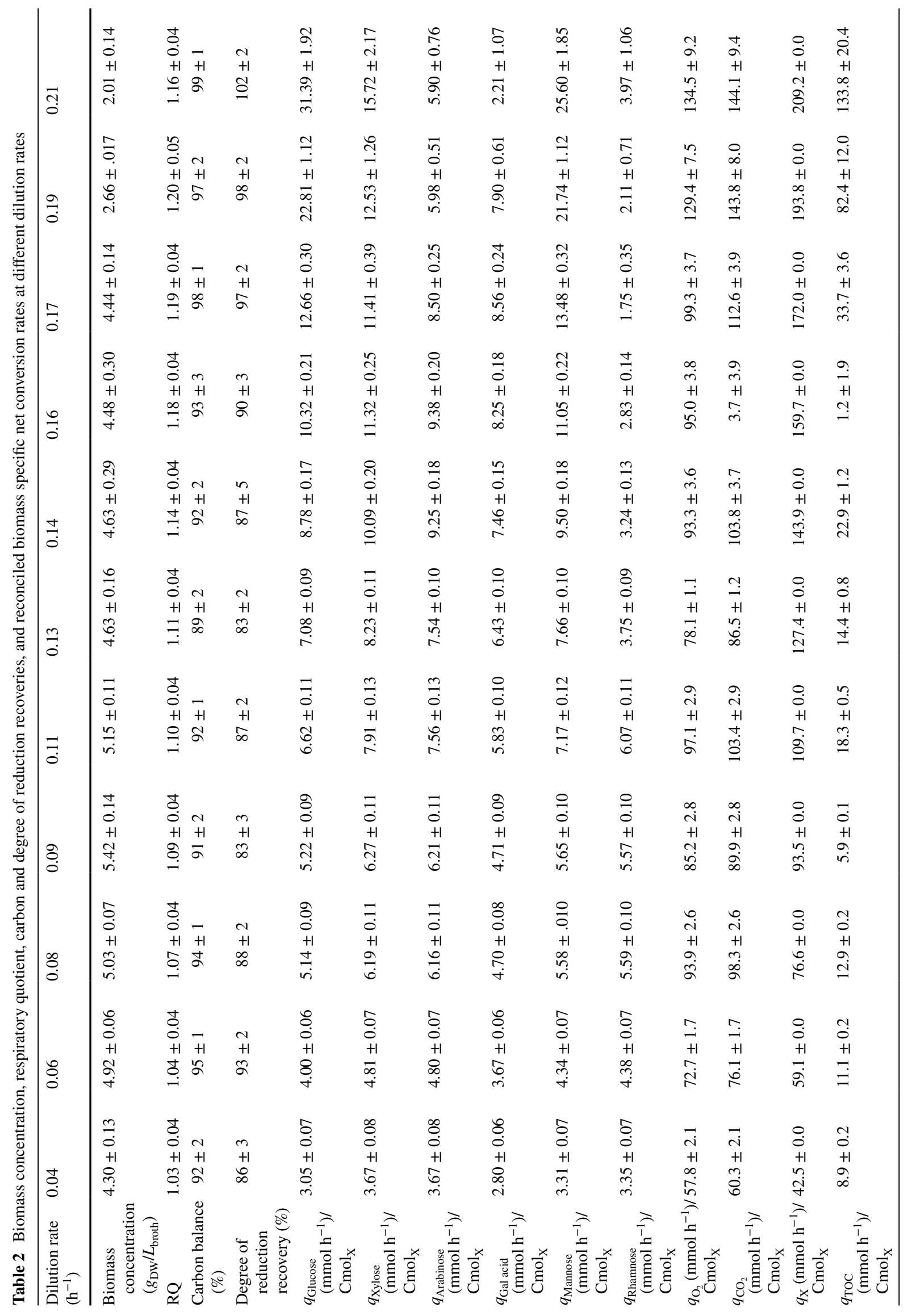


From the quantification of the residual concentrations of the carbon sources in the chemostat, it can be seen that during the first four steady states, up to a dilution rate of $0.093 \mathrm{~h}^{-1}$, all six carbon sources were consumed simultaneously (Fig. 4). From the fifth steady state $\left(D=0.11 \mathrm{~h}^{-1}\right)$, the residual concentrations of rhamnose, arabinose, and galacturonic acid started to increase, from $D=0.127 \mathrm{~h}^{-1}$ also xylose, from $D=0.16 \mathrm{~h}^{-1}$ mannose, and finally at $D=0.194 \mathrm{~h}^{-1}$ also glucose started accumulating.

At increasing dilution rate of the sequential chemostat fermentation, the increase of the residual concentrations of the different carbon sources occurred in the reverse order as the consumption of substrates in the multisubstrate batch fermentation. In these fermentations, L-rhamnose was only consumed after all other substrates were depleted, and appeared as the first increasing substrate at increasing dilution rate in the sequential chemostat cultivation. The observed increases of residual carbon-source concentrations also imply that the total amount of residual carbon source released from the chemostat via the effluent gradually increased with increasing dilution rate, and thus, the amount of consumed carbon gradually decreased. The result of this was that after an initial increase, the biomass specific respiration rate and steady-state biomass concentration levelled off, and finally decreased with increasing dilution rate (Fig. 5).

The observed increases of the residual concentrations of the different carbon sources indicate that the supply rate becomes higher than the uptake rate by the cells. In case of rhamnose, arabinose, galacturonic acid, and xylose, this already happened at biomass specific uptake rates of only $20 \%$ of the maximum value determined in the single substrate batch cultivations (see Tables 1, 2). In principle, this could have been caused by a very low affinity of the import systems for these substrates; however, it is to be expected that under carbon-limited chemostat conditions, high-affinity importers are expressed. Other possible explanations are catabolite repression and/or competition of multiple substrates for the same import system(s).

As mentioned above, glucose repression is likely to have occurred in the multisubstrate batch cultivations, as only after glucose was almost depleted the consumption of the other carbon sources started. In the carbon-limited chemostat cultivation, however, the residual glucose concentration was very low until a dilution rate of about $0.17 \mathrm{~h}^{-1}$ (Fig. 4). Nevertheless, the residual glucose concentration increased with increasing dilution rate (= growth rate) from $0.0125 \mathrm{mM}$ at the lowest growth rate of $0.043 \mathrm{~h}^{-1}$ to $1.4 \mathrm{mM}$ at the highest growth rate of $0.21 \mathrm{~h}^{-1}$ (Online Resource 6). If glucose repression would have been responsible for the limited uptake of the other carbon sources, this would imply that already at very low glucose concentrations repression of the uptake of the other substrates occurs. In [23], it was reported that in glucose limited chemostat cultivations of
Aspergillus nidulans, CreA-mediated carbon catabolite repression increased with increasing dilution rate and thus specific growth rate. However, it follows from chemostat theory that the residual concentration of the growth limiting substrate increases with increasing dilution rate [24]. It can, therefore, not be excluded that the observed CreA-mediated carbon catabolite repression at higher dilution rates observed by [23]. was in fact caused by the onset of glucose repression due to the increase of the residual glucose concentration.

As the last substrate which was depleted, before rhamnose consumption started in the multi substrate batch was arabinose (Fig. 3), and the second substrate appearing with rhamnose was also arabinose (Fig. 4), arabinose is a possible candidate repressor of L-rhamnose transport/metabolism. It has indeed been observed that in Aspergillus nidulans, the $\alpha$-L-rhamnosidase genes are strongly repressed by arabinose but also by glucose, ethanol, and sorbitol through a CreAindependent mechanism $[18,25]$. It might be that the uptake and/or metabolism of the other substrates (arabinose, galacturonic acid, xylose and mannose) is repressed by glucose only, via a CreA-dependent mechanism. However, from the results of the multi substrate batch cultivations it appears that also mannose represses the metabolism of xylose, arabinose, and galacturonic acid. A better zoom-in on the kinetics and putative catabolite repression systems is advanced in the following section.

\section{Substrate uptake kinetics}

To obtain a clearer picture on possible catabolite repression in A. niger, the biomass specific uptake rates of the different substrates in the sequential chemostat cultivations are plotted as a function of the residual substrate concentration (Fig. 6). In these plots, the maximum specific substrate uptake rate measured in the single substrate batch cultivations is indicated with a broken line. A Michaelis-Menten function (Eq. 3) was fitted to the data to obtain apparent $V_{\max }$ and $K_{\mathrm{m}}$ values for uptake of the different carbon sources in the multicarbon-source chemostat cultivation.

It can be seen from these plots that for all carbon sources, the maximum uptake rate is lower than observed in the single carbon-source batch cultivations. Especially, the uptake/ metabolism of galacturonic acid, arabinose, and rhamnose seems to be repressed, as at a certain point the uptake rates of these carbon sources level off far below their $q_{\mathrm{S}}{ }^{\max }$ values and subsequently decrease at increasing substrate concentration. In case of glucose, mannose, and xylose, repression does not seem to take place; however, with increasing substrate concentration, the specific substrate uptake does not reach the determined $q_{\mathrm{S}}{ }^{\max }$. A possibility could be that glucose, mannose, and xylose are taken up by the same transport systems, resulting in competition between those substrates for the same transporters. Until now, three glucose import 
Fig. 6 Biomass specific uptake rate of different substrates against residual substrate concentration during sequential chemostat cultivation. Maximum substrate uptake specific rate fitting using the MichaelisMenten equation
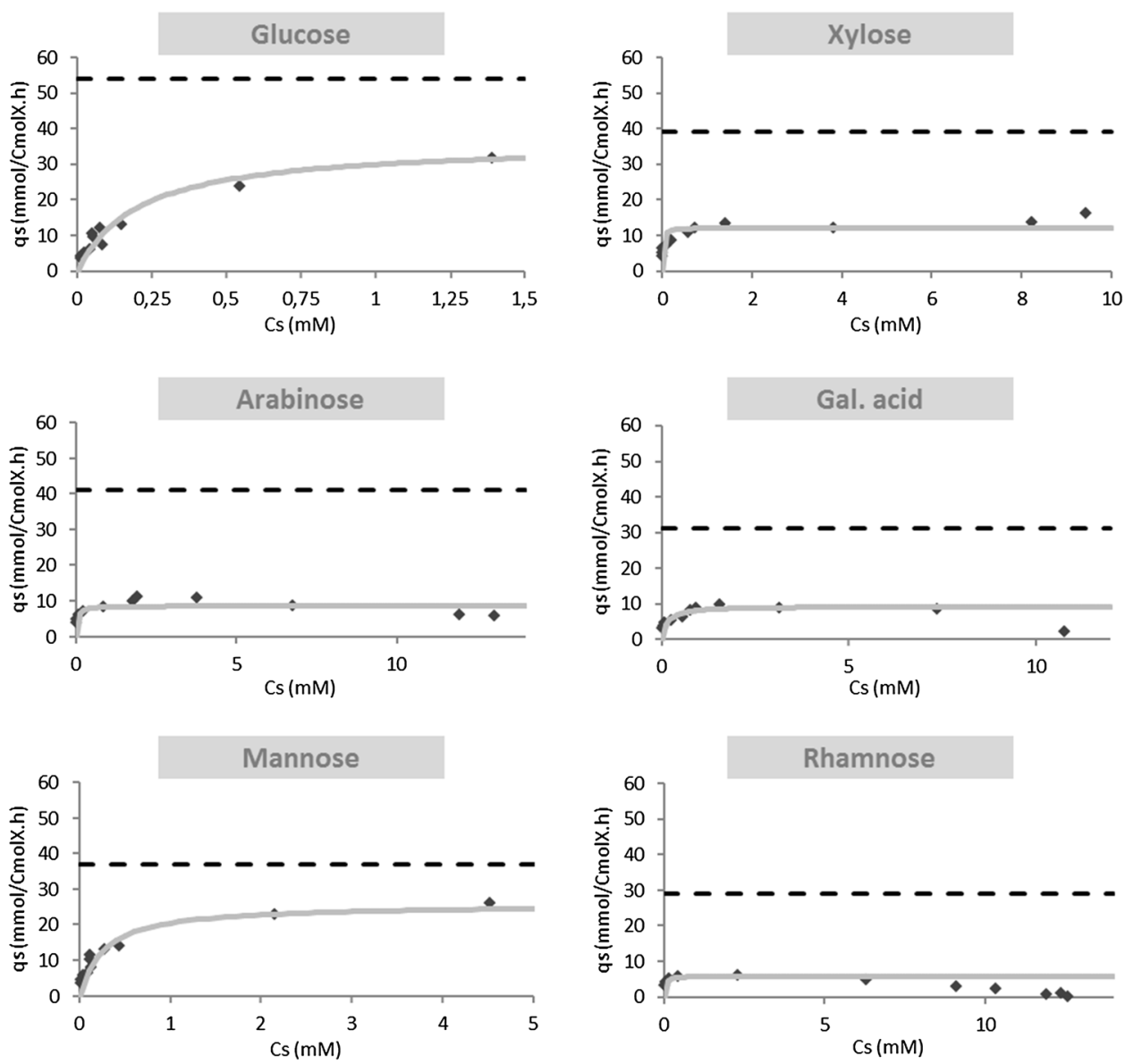

systems of A. niger have been characterized, namely, MSTA, MSTG, and MSTH. MSTA $\left(K_{\mathrm{m}, \text { glucose }} 0.025 \pm 0.010 \mathrm{mM}\right)$ also imports mannose $\left(K_{\mathrm{m}} 0.06 \pm 0.02 \mathrm{mM}\right)$ and xylose $\left(K_{\mathrm{m}}\right.$ $0.3 \pm 0.1 \mathrm{mM})$ [26]. MSTG $\left(K_{\mathrm{m}, \text { glucose }} 0.50 \pm 0.04 \mathrm{mM}\right)$, also imports mannose and possibly xylose, while MSTH ( $K_{\mathrm{m}, \text { glucose }} 0.06 \pm 0.005 \mathrm{mM}$ ) also imports mannose and fructose [27]. In addition, also three xylose import systems have been identified in A. niger, namely, XTLA, XTLB, and XTLC [18]. XTLA $\left(K_{\mathrm{m}} 0.09 \pm 0.03 \mathrm{mM}\right)$ also imports glucose $\left(K_{\mathrm{m}} 0.07 \pm 0.01 \mathrm{mM}\right)$ with the same $V_{\max }$ as for xylose. XTLB seems specific for xylose $\left(K_{\mathrm{m}} 15.0 \pm 4.50 \mathrm{mM}\right)$ and XTLC $\left(K_{\mathrm{m}} 4.71 \pm 1.04 \mathrm{mM}\right)$ imports glucose with a much higher affinity $\left(K_{\mathrm{m}} 0.11 \pm 0.02 \mathrm{mM}\right)$ and a roughly ten times higher $V_{\max }$ and should therefore be called a glucose importer.

From the published kinetic information on the six until now characterized sugar porters of $A$. niger, it appears that five of them are not very specific and import several sugars. In addition, in other filamentous fungi and yeasts, transport systems have been characterized that import several monosaccharides. In Aspergillus nidulans, the xylose transporter xtrD also imports glucose, galactose, and mannose [28], while the glucose transporters HxtB, HxtC, and $\mathrm{HxtE}$ also import fructose, mannose, and galactose [29]. A functional survey carried out by [30] found that the hexose transporter Hxt7 and galactose transporter GAL2 of Saccharomyces cerevisiae are able to import glucose, xylose, galactose, fructose, and mannose, while GAL2 in addition also imports ribose. Similar results were obtained for the sugar importers GXF1 and GXS1 of Candida intermedia, DEHA0D02167 and XylHP of Debaryomyces hansenii, and XUTt1 and XUT3 of Scheffersomyces stipites.

To model the uptake of glucose, mannose, and xylose, we assumed that these sugars are taken up by the same import systems and that their uptake could be described by competition (Eq. 4).

Both the specific uptake rates of glucose and mannose could be described well with this equation. For the specific uptake of xylose, no good fit could be obtained, indicating that besides competition probably also catabolite repression of xylose uptake and/or metabolism occurred (Online Resource 7).

The switch function (Eq. 5) was used to model glucose repression of the uptake of xylose, arabinose, galacturonic acid, and rhamnose in the sequential chemostat cultivation. The specific uptake rates of xylose, arabinose, and galacturonic acid could be described reasonably well by glucose repression (Online Resource 8); however, no fit could be 
obtained for the uptake of rhamnose (result not shown). As it is known that in A. nidulans, the $\alpha$-L-rhamnosidase genes are strongly repressed by arabinose [23], and the same switch function (Eq. 5) was used to describe the repression of rhamnose uptake/metabolism by arabinose, which gave a reasonable fit (Online Resource 8). Further experiments using simultaneous substrate cultivation, i.e., with and without competitor/repressing substrates, would be advantageous to validate the mentioned hypothesis.

\section{Conclusions}

Large differences in $q_{\mathrm{S}}{ }^{\max }$ and $\mu^{\max }$ for individual substrates was observed, but in combination, and some carbon sources were consumed simultaneously and some sequentially. We found that the uptake of glucose, xylose, and mannose seems to be competing for the same transport systems, while the uptake of arabinose, galacturonic acid, and rhamnose appeared to be repressed by the presence of other substrates.

The stoichiometric and kinetic characterization of growth on different carbon sources, and the role of substrates as repressors or competitors during cultivation, should be considered in the design of a fermentation process based on plant waste feedstocks. In addition, the transition from simple carbon sources to more complex substrate mixtures requires careful attention.

Acknowledgements The authors would like to thank Johan Knoll and Reza Maleki Seifar for analytical support; Simon Felz for partly developing the analytical platform in HPIC system; Ruud van Tol and Marcel Langeveld for construction of the second sampling device.

\section{Compliance with ethical standards}

Funding This work was supported by the BE-Basic foundation (bebasic.org).

Conflict of interest The authors declare that they have no conflict of interest.

Open Access This article is distributed under the terms of the Creative Commons Attribution 4.0 International License (http://creativecommons.org/licenses/by/4.0/), which permits unrestricted use, distribution, and reproduction in any medium, provided you give appropriate credit to the original author(s) and the source, provide a link to the Creative Commons license, and indicate if changes were made.

\section{References}

1. Lin Y, Tanaka S (2006) Ethanol fermentation from biomass resources: current state and prospects. Appl Microbiol Biotechnol 69:627-642
2. Rumbold K, van Buijsen HJJ, Overkamp KM et al (2009) Microbial production host selection for converting second-generation feedstocks into bioproducts. Microb Cell Fact 8:64

3. Gouka RJ, Punt PJ, Van Den Hondel CA (1997) Efficient production of secreted proteins by Aspergillus: progress, limitations and prospects. Appl Microbiol Biotechnol 47:1-11

4. de Souza WR, de Gouvea PF, Savoldi M et al (2011) Transcriptome analysis of Aspergillus niger grown on sugarcane bagasse. Biotechnol Biofuels 4:40

5. van Maris AJA, Abbott DA, Bellissimi E et al (2006) Alcoholic fermentation of carbon sources in biomass hydrolysates by Saccharomyces cerevisiae: current status. Antonie van Leeuwenhoek Int J Gen Mol Microbiol 90:391-418

6. Moses V, Prevost C (1966) Catabolite repression of B-galactosidase synthesis in Escherichia coli. Biochem J 100:336-352

7. Ronne H (1995) Glucose repression in fungi. Trends genet 11:12-17

8. Lameiras F, Heijnen JJ, van Gulik WM (2015) Development of tools for quantitative intracellular metabolomics of Aspergillus niger chemostat cultures. Metabolomics 11:1253-1264

9. Ruijter GJ, van de Vondervoort PJ, Visser J (1999) Oxalic acid production by Aspergillus niger: an oxalate-non-producing mutant produces citric acid at $\mathrm{pH} 5$ and in the presence of manganese. Microbiology 145:2569-2576

10. Sloothaak J, Schilders M, Schaap PJ, de Graaff LH (2014) Overexpression of the Aspergillus niger GatA transporter leads to preferential use of D-galacturonic acid over D-xylose. AMB Express 4:66

11. Vishniac W, Santer M (1957) The thiobacilli. Bacteriol Rev 21:195-213

12. Mashego MR, Rumbold K, De Mey M et al (2007) Microbial metabolomics: past, present and future methodologies. Biotechnol Lett 29:1-16

13. Niedenführ S, ten Pierick A, van Dam PTN et al (2016) Natural isotope correction of MS/MS measurements for metabolomics and 13C fluxomics. Biotechnol Bioeng 113:1137-1147

14. Cruz ALB, Verbon AJ, Geurink LJ et al (2012) Use of sequentialbatch fermentations to characterize the impact of mild hypothermic temperatures on the anaerobic stoichiometry and kinetics of Saccharomyces cerevisiae. Biotechnol Bioeng 109:1735-1744

15. Verheijen PJT (2010) Data reconciliation and error detection. Balanc React Model

16. Schäuble S, Stavrum AK, Puntervoll P et al (2013) Effect of substrate competition in kinetic models of metabolic networks. FEBS Lett 587:2818-2824

17. Fries N, Kallstromer L (1965) A requirement for Biotin in Aspergillus niger when grown on a rhamnose medium at high temperature. Physiol Plant 18:191-200

18. Sloothaak J, Tamayo-Ramos JA, Odoni DI et al (2016) Identification and functional characterization of novel xylose transporters from the cell factories Aspergillus niger and Trichoderma reesei. Biotechnol Biofuels 9:148

19. Roels JA (1983) Energetics and kinetics in biotechnology. Elsevier Biomedical Press BV, Amsterdam

20. Flipphi M, Van de Vondervoort PJI, Ruijter GJG et al (2003) Onset of carbon catabolite repression in Aspergillus nidulans: parallel involvement of hexokinase and glucokinase in sugar signaling. $\mathrm{J}$ Biol Chem 278:11849-11857

21. Schneider RP, Wiley WR (1971) Regulation of sugar transport in Neurospora crassa. J Bacteriol 106:487-492

22. Portnoy T, Margeot A, Linke R et al (2011) The CRE1 carbon catabolite repressor of the fungus Trichoderma reesei: a master regulator of carbon assimilation. BMC Genom 12:269

23. Ilyes H, Fekete E, Karaffa L et al (2004) CreA-mediated carbon catabolite repression of beta-galactosidase formation in 
Aspergillus nidulans in growth rate dependent. FEMS Micobiol Lett 235(1):147-151

24. Herbert D, Elsworth R, Telling RC (1956) The continuous culture of bacteria; a theoretical and experimental study. J Gen Microbial 14:601-622

25. Tamayo-Ramos JA, Flipphi M, Pardo E et al (2012) L-Rhamnose induction of Aspergillus nidulans $\alpha$-L-rhamnosidase genes is glucose repressed via a CreA-independent mechanism acting at the level of inducer uptake. Microb Cell Fact 11:26

26. van Kuyk PA, Diderich JA, MacCabe AP et al (2004) Aspergillus niger mstA encodes a high-affinity sugar/ $\mathrm{H}+$ symporter which is regulated in response to extracellular pH. Biochem J 379:375-383
27. Sloothaak J, Odoni DI, de Graaff LH et al (2015) Aspergillus niger membrane-associated proteome analysis for the identification of glucose transporters. Biotechnol Biofuels 8:150

28. Colabardini AC, Ries LNA, Brown NA et al (2014) Functional characterization of a xylose transporter in Aspergillus nidulans. Biotechnol Biofuels 7:46

29. dos Reis TF, de Lima PBA, Parachin NS et al (2013) Identification and characterization of putative xylose and cellobiose transporters in Aspergillus nidulans. Biotechnol Biofuels 9:204

30. Young E, Poucher A, Comer A et al (2011) Functional survey for heterologous sugar transport proteins using Saccharomyces cerevisie as a host. App Environ Microbiol 77:10 